\title{
Anomalous Shift of the Recombination Energy in Single Asymmetric Quantum Wells
}

\author{
N.O. Dantas ${ }^{a}$, Fanyao $\mathrm{Qu}^{a}$, and P.C. Morais ${ }^{b}$ \\ ${ }^{a}$ Universidade Federal de Uberlândia, Faculdade de Física, CEP 38400-902, Uberlândia-MG, Brazil \\ ${ }^{b}$ Universidade de Brasília, Instituto de Física, Núcleo de Física Aplicada \\ CEP 70919-970, Brasília-DF, Brazil
}

Received on 23 April, 2001

\begin{abstract}
Self-consistent numerical calculation and photoluminescence (PL) measurements have been used to investigate the temperature dependence of the optical Stark effect in $n$-doped GaAs/AlGaAs single asymmetric quantum wells (SAQWs), grown by molecular beam epitaxy. In the low-temperature regime $(5$ to $40 \mathrm{~K})$ a remarkable blue shift $(9.8 \mathrm{meV})$ is observed in the PL peak energy, as the optical excitation intensity increases from 0.03 to $90 \mathrm{~W} / \mathrm{cm}^{2}$. The blue shift is well explained by the reduction of the two-dimensional electron gas (2DEG) density, due to a charge-transfer mechanism. At about $80 \mathrm{~K}$, however, an anomalous behavior of the PL peak energy was found, i.e. a red shift has been observed as the optical excitation intensity increases. This anomalous behavior has been explained by combining the effects of band gap renormalization, band bending, temperature dependence of the band gap, temperature dependence of the 2DEG density, and temperature dependence of the fundamental energy position.
\end{abstract}

\section{Introduction}

One-side modulation-doped or single asymmetric quantum wells (SAQWs) have been used as ideal systems to investigate different aspects related to many-body effects in two-dimensional (2D), one-component carrier plasmas [1]. The SAQW is a special case of modulationdoped quantum well (MDQW) where the remote doping is made only in one side of the single quantum well. The typical growth profile of a GaAs/AlGaAs SAQW includes a thick GaAs buffer layer on top of the GaAs substrate, a short-period GaAs/AlGaAs superlattice, the GaAs SAQW, the undoped AlGaAs spacer layer, the $n$ - or $p$-type doped AlGaAs layer, and finally the GaAs cap layer. Among the many-body effects investigated using SAQWs are the Fermi-edge singularity (FES) [2], the band gap renormalization [3], and dimensionality crossover [4], all of them strongly dependent upon the carrier temperature and their density $\left(N_{S}\right)$. For instance, the low temperature and high carrier concentration favor the FES observation in PL experiments, while FES would be smeared out by high temperature and low carrier density. Besides FES, band gap renormalization also strongly depends upon the carrier density, $N_{S}$, according to $\left(N_{S}\right)^{\eta}$. The exponent $\eta$ is related to the dimensionality of the system, being $\eta=1 / 2$ for three-dimensional (3D) carrier plasma and $\eta=1 / 3$ for $2 \mathrm{D}$ carrier plasma. At this point we empha- size the great interest of using SAQWs to investigate many-body effects in 2D semiconductor heterostructures, once the carrier gas density in such structure can be continuously varied from its maximum value down to zero. Continuous change of the $2 \mathrm{D}$ carrier density has been achieved by applying a bias voltage in a field effect transistor configuration [5] - electric Stark effect. Alternatively, continuous change of the $2 \mathrm{D}$ carrier density has been obtained through illumination of the sample with increasing optical excitation intensity $(P)$ - optical Stark effect. The signature of the optical Stark effect is a remarkable energy shift (typically $10 \mathrm{meV}$ ) in the fundamental optical transition. The explanation of the PL blue shift with the increase of the laser excitation intensity was first put forward by Chaves et al. [6]. Thereupon, it was further confirmed by following works [3]. The established optical control mechanism used to explain the experiment requires illumination with photons having energy higher than the band gap of the undoped spacer layer. The attention is focused on the electron-hole pairs photocreated in the spacer layer. In the n-type doped structure, the photocreated electron initially moves back to the spatially distant donors while the photocreated hole is drifted into the quantum well by the built-in electric field, there recombining with $2 \mathrm{D}$ electrons. Later on, the photocreated electron moves from the donor into the quantum well by tunneling through the spacer layer. At a given opti- 
cal excitation intensity the steady-state 2DEG density is lower than the density $\left(N_{S}^{0}\right)$ at or near zero excitation. Although the charge-transfer mechanism has been successfully used to explain the experimental, all the reported studies concerning the optical Stark effect have been performed at low-temperatures. However, the influence of finite temperatures on the optical Stark effect is obviously important for both applied purposes and fundamental physics. Nevertheless, the temperature dependence of optical Stark effect has not been investigated yet, in spite of the huge technological impact of the micro-electronic-optical devices and optical communications, both operating at finite temperatures. Investigation of the effects of finite temperatures upon the optical Stark effect is the main issue of the present study.

\section{Experimental details and re- sults}

The PL experiments reported in this study were carried out using two GaAs/ $\mathrm{Al}_{0.35} \mathrm{Ga}_{0.65}$ As SAQW samples (W1506 and W1413), grown by molecular beam epitaxy. Sample W1506 consists of a semi-insulating GaAs substrate followed by a $1.3 \mu \mathrm{m}$-thick GaAs buffer layer, a GaAs $/ \mathrm{Al}_{0.35} \mathrm{Ga}_{0.65}$ As superlattice (typically $7 \times 35 \AA$ $+8 \times 100 \AA$ ), and by the nominally $152 \AA$-thick GaAs SAQW. The 2D electron gas (2DEG) inside the GaAs SAQW is a result of the electron-transfer, through a $295 \AA$-thick undoped $\mathrm{Al}_{0.35} \mathrm{Ga}_{0.65} \mathrm{As}$ spacer layer, from a $385 \AA$ Si-doped $\mathrm{Al}_{0.35} \mathrm{Ga}_{0.65}$ As layer. A $250 \AA$-thick GaAs cap layer was grown on top of the overall structure. Samples W1413 and W1506 have similar growth profiles. In the W1413 sample, the asymmetric quantum well width is $150 \AA$, the thickness of spacer layer is $300 \AA$, and the thickness of the Si-doped $\mathrm{Al}_{0.35} \mathrm{Ga}_{0.65} \mathrm{As}$ barrier region is $380 \AA$. Both samples have electron mobility higher than $4 \times 10^{4} \mathrm{~cm}^{2} / \mathrm{V} \times \mathrm{s}$ at liquid Helium temperature. Samples were optically excited above the band gap of the undoped $\mathrm{Al}_{0.35} \mathrm{Ga}_{0.65} \mathrm{As}$ spacer layer, using the $5145 \AA$ argon-ion laser line. The PL spectra were recorded using a SPEX-750M monochromator equipped with a Joban-Yvon CCD $2000 \times 800-3$. The NEOCERA LTC-11 temperature controller was used during the PL measurements.

Fig. 1 shows the PL spectra of sample W1506 under $P=0.4 \mathrm{~W} / \mathrm{cm}^{2}$, at different temperatures $(10,30,50$, and $80 \mathrm{~K}$ ). The PL peak-A, located at $1521.7 \mathrm{meV}$ (10 $\mathrm{K})$, is associated to the $\Delta n=0$ transition, i.e. between the ground state conduction subband to the ground state heavy-hole subband of the GaAs SAQW. As the optical excitation intensity increases the 2DEG density decreases due to the electron-transfer mechanism. At about $P=90 \mathrm{~W} / \mathrm{cm}^{2}$ all the electrons are removed out from the GaAs SAQW. Then, the electron-transfer turns the $152 \AA$-thick GaAs SAQW into an almost perfect square potential well. In this case, the calculated $\Delta n=0$ recombination energy is $1538.7 \mathrm{meV}$ and the exciton binding energy associated to the ground state in the GaAs SAQW is $8.2 \mathrm{meV}$. Thus, a free exciton transition in a square GaAs potential well would be expected to occur at about $1530.5 \mathrm{meV}$. Note the good agreement between the calculated value (1530.5 meV) and the observed PL peak-A position (1531.7 meV) at higher optical excitation intensity $\left(P=90 \mathrm{~W} / \mathrm{cm}^{2}\right)$.

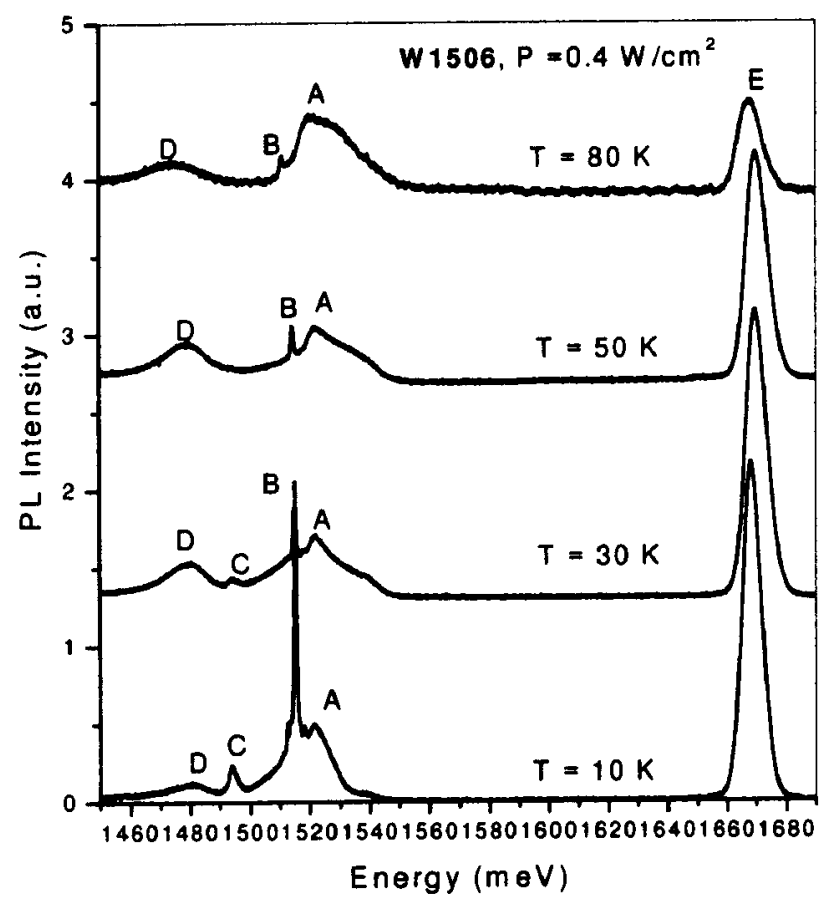

Figure 1. PL spectra of sample W1506 under $P=0.4$ $\mathrm{W} / \mathrm{cm}^{2}$, at different temperatures $(10,30,50$, and $80 \mathrm{~K})$.

The PL peak-B, located at $1514.8 \mathrm{meV}(10 \mathrm{~K})$, is attributed to the recombination from the thick GaAs buffer layer. It is worthy to mention that the PL peaks $\mathrm{A}$ and $\mathrm{B}$ are very much close together in the weak optical excitation intensity regime, because of the strong band bending and band gap renormalization effects. However, as $P$ increases the PL peak-A tends to separate from the PL peak-B. Finally, they are completely separated at the higher optical excitation intensity regime. Moreover, PL peaks A and B demonstrate quite different temperature dependence. As expected, PL peak-B shows normal temperature dependence, once intensity and energy peak position decrease monotonically with the increase in temperature. In contrast, PL peak-A experiences a different behavior once its intensity with respect to the $\mathrm{PL}$ peak-B intensity increases monotonically, as the temperature increases. The PL peak-C (1493.8 meV) is assigned to the carbon acceptor transition in the GaAs substrate. PL peaks $\mathrm{C}$ (1493.8 meV) and D (1480.1 meV) follow similar behav- 
ior, as far as the increase of the optical excitation intensity is concerned. In contrast, PL peaks $\mathrm{C}$ and D show different temperature dependence. Thus, the PL peak $\mathrm{D}$ may originate from the residual acceptor transition in the nominally undoped GaAs SAQW, accompanied by LO-phonon emission. Finally, PL peak E (1668.7 $\mathrm{meV}$ ) has its position almost independent of the optical excitation intensity, being associated to the interband transition from the short-period $\mathrm{GaAs} / \mathrm{Al}_{0.35} \mathrm{Ga}_{0.65} \mathrm{As}$ superlattice.

Fig. 2 shows the optical excitation intensity dependence of the recombination energy of the W1413 sample, at different temperatures. At low temperature $(10 \mathrm{~K})$, a strong blue shift in recombination energy, as the optical excitation intensity increases, is found. As the temperature increases from $10 \mathrm{~K}$ to $40 \mathrm{~K}$, the blue shift in recombination energy is still observed, though reduced from $9.2 \mathrm{meV}(10 \mathrm{~K})$ to $8.4 \mathrm{meV}(40 \mathrm{~K})$. As the temperature is further increased a breakdown of the blue shift is observed, as shown by the open circles ( 80 $\mathrm{K}$ ) in Fig. 2. Above about $80 \mathrm{~K}$ the recombination energy decreases monotonically as the temperature increases. At $100 \mathrm{~K}$, a slight blue shift ( $2 \mathrm{meV}$ ) has been found.

\section{Discussion}

At very low temperature, residual acceptor states play a key role in the optical properties of intentionally donordoped semiconductor materials. Under such condition few acceptor states affect the position of the Fermi energy, for electrons from donors get captured at acceptor sites, resulting in some ionized donors. Under low optical excitation intensity, the photogenerated excess holes collected by the SAQW are less than the number of the ionized acceptors introduced by the residual doping. Then, the ionized residual acceptors rapidly localize photoholes. Since in $k$-space the state of the localized holes is extended, recombination of all occupied conduction-band states with different $\boldsymbol{k}$, from 0 up to the Fermi wave vector, $\boldsymbol{k}_{F}$, and localized holes is allowed. At a suitable acceptor concentration the restriction of the $k$-selection rule imposed on the recombination process is relaxed. So, at low temperature, the free photoelectron-localized-hole transitions dominate the optical emission process. Since the trapping of holes by electrons is very efficient, the optical transitions remain $k$-nonconserving. As a consequence, in SAQWs, low-temperature PL spectra present broad and step-like bands with sharp edges at the effective band gap and at the Fermi energy cutoff (see Fig. 1).

In SAQWs, the optical Stark effect clearly shows three different behaviors as a function of both optical excitation intensity and temperature (see Fig. 2). First, at low optical excitation intensity, and in the temperature range from 5 to $100 \mathrm{~K}$, an anomalous tempera- ture dependence of the PL recombination energy is observed. As the temperature increases from 5 to $100 \mathrm{~K}$ the PL peak energy moves upwards to a peak value, at intermediate temperatures, to further come down at the high-temperature end (100 K). Second, at high optical excitation intensity, the PL peak energy associated to the SAQW presents normal temperature dependence, i.e. the PL peak energy moves monotonically to lower energy as the temperature increases from 5 to $100 \mathrm{~K}$. Third, a continuous transition between the two different regimes has been observed at intermediate values of optical excitation intensity.

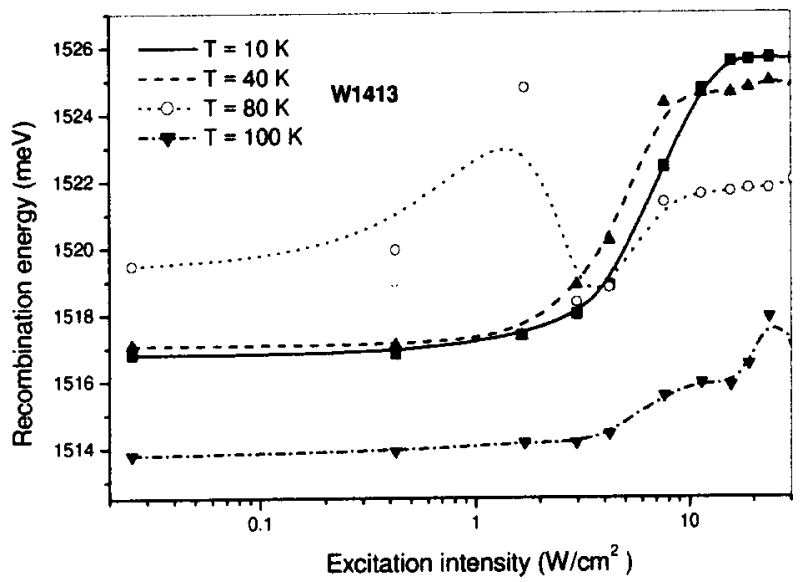

Figure 2. Recombination energy as a function of the optical excitation intensity, for sample $\mathrm{W} 1413$, at $\mathrm{T}=10 \mathrm{~K}$ (open squares), $\mathrm{T}=40 \mathrm{~K}$ (solid up triangles), $\mathrm{T}=80 \mathrm{~K}$ (open circles), and (solid down triangles). The lines are just guides for the eye.

To understand the complex features in the optical spectra of SAQWs, particularly their dependence upon the optical excitation intensity and temperature, the combined effects of band gap energy shrinkage, band bending, band gap renormalization, temperature dependence of the 2DEG, and relaxation of ionized acceptors should be taken into account. This means that a quantitative description of the temperature dependence of both 2DEG density and ground state energy has to be performed. Therefore, self-consistent calculation, which numerically solves the coupled Schrödinger and Poisson equations, has been carried out for sample W1413. The correspondent result will be published in a forthcoming paper. The following parameters have been used in the calculation: donor bound energy fixed at $E_{D}=50 \mathrm{meV}$, low frequency dielectric constant in the well $\left(\varepsilon_{W}=13.2 \varepsilon_{0}\right)$ and in the barrier $\left(\varepsilon_{B}=12.2 \varepsilon_{0}\right)$, and effective electron mass in the well $\left(m_{e}^{W}=0.067 m_{0}\right)$ and in the barrier $\left(m_{e}^{B}=0.092 m_{0}\right)$. It is found that the electron areal density increases as the temperature increases. In addition, the higher the donor concentration the larger the electron areal density and its increasing rate. Reduction of the electron fundamental energy due to the temperature increase was about $1 \mathrm{meV}$. Ac- 
tually, in the low optical excitation intensity regime, the blue shift due to the electron-transfer mechanism is negligible. As the temperature increases thermal effects tend to spread the 2DEG over the full density of states. Then, the PL peak energy shifts toward higher values. On the other side, as the temperature increases, the shrinkage of the band gap and the reduction of the ground state energy lead to a red shift of the PL peak energy. Reduction of the PL peak energy, due to the shrinkage of the band gap energy, is about $8.5 \mathrm{meV}$ (0.69 meV) as the temperature increases from 40 to 80 $\mathrm{K}(10$ to $40 \mathrm{~K})$. Then, in the low optical excitation regime and below $80 \mathrm{~K}$, the resultant effect leads to a blue shift in the PL peak energy. With further increase in temperature (above $80 \mathrm{~K}$ ), the shrinkage of the band gap becomes dominant $(12.1 \mathrm{meV}$ in the temperature range of 80 to $100 \mathrm{~K}$ ), leading to a red shift in the $\mathrm{PL}$ peak energy. At intermediate values of optical excitation intensities, however, the electron-transfer mechanism governs the optical emission process, reducing the 2DEG density in the SAQW. The band bending and the band gap renormalization decrease as the optical excitation intensity increases. The PL peak energy is expected to move toward higher energy values. Eventually, in the high optical excitation intensity regime, all electrons are removed out from the SAQW by the photogenerated holes. The SAQW turns into a perfect square potential well. Then, as expected, normal temperature dependence of the PL recombination energy as well as symmetric PL spectra are observed. In other words, the anomalous dependence of the PL recombination energy is smeared out as the optical excitation intensity increases. This feature supports the picture of the competition between the optical Stark effect and the electron-transfer mechanism in explaining the optical properties of SAQWs, at finite temperatures and in a wide range of optical excitation intensities.

In summary, the band gap renormalization, band bending, temperature dependence of the 2DEG density, and temperature dependence of the fundamental energy position are all coupled together to determine the very complex behavior of the SAQW PL peak position.

\section{Acknowledgments}

Support from the Brazilian agencies FAPMIG, FAP$\mathrm{DF}$, and $\mathrm{CNPq}$ is gratefully acknowledged.

\section{References}

[1] P. Hawrylak, Phys. Rev. B 44, 6262 (1991).

[2] Fanyao Qu, N.O. Dantas, and P.C. Morais, Europhys. Lett. 53, 790 (2001).

[3] A.J.C. Cardoso, Fanyao Qu and P.C. Morais, Phys. Rev. B 60, 4501 (1999).

[4] Fanyao Qu and P.C. Morais, IEEE J. Quantum Electron. 36, 348 (2000).

[5] C. Delalande, J.A. Brum, G. Bastard, Solid State Commun. 59, 613 (1986).

[6] A.S. Chaves, A.F.S. Penna, J.M. Worlock, G. Weimann, and W. Schlapp, Surf. Sci. 170, 618 (1986). 